\title{
Ridesharing in Muscat: Can it be a Sustainable Solution for the Traffic Congestion?
}

\author{
C. Triki ${ }^{*}, 1$, A. Al-Maimani ${ }^{2}$, J. Akil ${ }^{3}$
}

\begin{abstract}
We deal with developing a Decision Support System (DSS) to promote the ridesharing among both students and staff of a big organization. The DSS includes a set of functions that allow the management of the riders' requests and drivers' availability and embeds a novel two-phase optimization approach that helps in defining the optimal riders-drivers matching. The first phase consists of solving a constraint programming model that generates all the feasible routes. Then, the second phase a bin packing based model is solved to find the optimal route for every driver in order to serve the set of riders assigned to her vehicle. We conclude by an illustrative example that shows the validity of our DSS and, finally, by a discussion on the possible commercialization of such a platform.
\end{abstract}

Keywords: Ridesharing, Matching problem, Bin-packing problem

Manuscript was received on 19/08/2017, revised on 17/11/2017 and accepted for publication on 23/11/2017.

\section{Introduction}

Traffic jam is becoming a real issue in Muscat, the capital and biggest city in the Sultanate of Oman. Indeed, with a growth rate of more than 9\% per year (World Population Review, 2017), the population of Oman is estimated to be 4,737,861 inhabitants with nearly $50 \%$ of the population living in Muscat Governorate. Consequently, the population growth is making a high pressure on the existing infrastructure of the city, leading to always growing traffic congestion. Moreover, urban public transportation is scarcely developed since the first public urban bus service has been inaugurated only in 2015, and there is no train or metro service network connecting the wide area of Muscat. In this context, private cars provide more convenience and flexibility for road users. Nonetheless, the total number of vehicles is not compatible with the available transport infrastructure, resulting in occasional heavy traffic jams, especially at peak hours.

Road congestions can be explained by "the tragedy of the commons" theory (Hardin, 1968). When applied to our case study, this theory illustrates a situation in which public goods, the roads in our case, are shared by self-interest agents keen to maximize the utility. The agents, generally, do not adopt cooperative strategies since each person thinks that the others, including public authorities, should do the necessary action to solve the problem of traffic congestion.

\footnotetext{
* Corresponding Author.

${ }^{1}$ Department of Mechanical and Industrial Engineering, Sultan Qaboos University, Oman and Department of Engineering for Innovation, University of Salento, Italy. Email: chefi@squ.edu.om.

2 Department of Mechanical and Industrial Engineering, Sultan Qaboos University, Oman. Email: a.maimani@almuhandisgroup.com.

${ }^{3}$ Department of Natural Resource Economics, Sultan Qaboos University, Oman. Email: akil@ squ.edu.om.
} 
This leads to an inefficient way of using scarce resources such as time and energy. If we leave aside the cost of energy paid uselessly by each agent, the daily time lost by road users in traffic congestions has an opportunity cost and the air pollution provoked by an excessive number of cars in circulation has a considerable cost for the whole community, presently and for future generations as well. Consequently, traffic congestion is a serious issue contrasting with economic efficiency.

In the literature, several remedies have been suggested to reduce the aversive effects of traffic congestion such as pricing methods and ridesharing. An example of such a mechanism has been proposed in China where the approximate annual loss caused by traffic congestion and accidents is estimated to be 170 billion Yuan (Zhu et al., 2008). The authors suggested the intervention of "road managers" to apply a toll payment for reducing bottlenecks at the peak hours. However, they did not specify the payment mechanism and did not discuss how to determine the toll amount. This is a crucial factor since high amounts will favor high-income users to use the roads in peak hours, whereas low toll amounts will not have any effect on dissuading drivers to use their private cars.

On the other hand, ridesharing is defined as a cooperative strategy between actors and it is privileged as a sustainable solution to reduce the use of private cars and hence reduce the associated externalities. In fact, exploiting the empty seats within a shared car might combine the convenience of using a private car but at the same time contributing to reduce road congestions. This solution has been proposed by a wide number of researchers belonging to multidisciplinary research fields such as socio-economy (Brownstone and Golob, 1992), transportation (Morency, 2007), operations research (Agatz et al., 2012) and artificial intelligence (Kleiner et al., 2011).

In reality, car ridesharing has been widely diffused in USA and Europe since 2011. Taking advantage of the wide availability of smartphone technologies, real time car ridesharing applications were developed to allow connecting demand and supply for rides, hence reducing waiting time. Real-life applications show that ridesharing is a valid solution that can compete with conventional cable systems by reducing not only waiting time but also traveling cost.

In the next section, we will review the state of the art in the existing literature and in business platforms operating in ridesharing market. The payment systems adopted by some of the available platforms will be highlighted. Our focus will be on developing an optimal matching and an efficient payment scheme that might represent the main economic incentive that encourages both passengers and car owners to change their behaviors. Finally, a case study related to Sultan Qaboos University is implemented and tested through a server platform based on the road network of the city of Muscat (a preliminary and simplified version of this work appeared in a conference proceedings by Al-Riyami et al., 2016).

\section{Literature Review}

According to Furuhata et al. (2013) and Chan and Shaheen (2012), traffic congestion reduces the quality of life of road users and sharing the empty seats in private cars can be a solution to mitigate traffic circulation problems. In practice, ridesharing is a system that can 
lead, in cooperation with road users, to overcome the discomfort of traffic congestion and may allow not only to enjoy the comfort of using private cars but also to reduce the travel cost. Furthermore, ridesharing has positive externalities such as decreasing traffic congestion, fuel consumption, and the consequent air pollution.

Kleiner et al. (2011) identified three conditions necessary for a ridesharing system to be efficient: flexibility, high availability and adequate incentive. Apparently, if users did not succeed to get a ride within a few attempts, they will be dissuaded to use it furthermore and if the drivers are not motivated by a high profit, only a few drivers will be willing to share their cars.

Nowadays, many ridesharing platforms are operating around the world; those who had wide success include ZimCar, Uber and Lift. The pricing mechanism of these big businesses is competitive with traditional taxi systems and cable services but at peak hours, holidays or late hours at night higher rates or surcharge prices are applied. This behavior has in general a dissuasive impact since surcharge prices are often considered to be unfair by riders.

In general, in most applications, credit cards and cash payments are used and the travel cost is computed based on the following factors:

- Distance (total miles traveled)

- Time (total minutes traveled)

- $\quad$ Base Charge (the amount the ride starts at)

- $\quad$ Service Fee (added to each ride charge)

- Time Surcharge (a percentage added during the busiest times or peak hours)

Indeed, the existing ridesharing systems are mostly based on fixed assignments to optimize the travelled distance (Kleiner, 2009). Moreover, some studies have developed pricing models to ensure fair payments to users. In particular, Kamar and Horvitz (2009) suggested the ABC dynamic ridesharing system to ensure fair payments using a VCG mechanism as incentives for riders and drivers (Nisan et al., 2007; Kleiner et al., 2011). On the other hand, new applications are focused on using the auction approach that gives the opportunity to riders to bid for a ride whereas drivers can select the highest bid (Kleiner et al., 2011). Furthermore, a recent study (Asghari et al., 2016) developed a model in which drivers bid in real time on requests and the server determines the highest bidder and assigns the rider to that driver. The latter model is automated since the matching between demand and supply of rides is based on the predefined profile of the riders who express not only their willingness to pay for a trip but also their willingness to accept some detours in order to receive an explicit discount rate. On the other hand, driver's profile shows her expected cost, which is a function of distance and time. After matching demand and supply bids, the application assigns the ride to the driver whose bid is maximizing the platform profit.

This mechanism is comparable with the model used by the existing platforms, Bidride and GabbyGo, in which drivers have to bid for a specific ride request and the rider then decides which bid to accept based on several factors such as price, travel time, driver rating or type of car. However, while Asghari et al. (2016) suggested that the server decide which rider to select, in the Bidride and GabbyGo platforms, the rider, comparing drivers' bids, has the opportunity to decide which car to share. Furthermore, while Asghari et al.'s model seems giving more 
advantage to the provider maximizing his profit, Bidride and GabbyGo are probably favoring riders giving them the opportunity to select the driver who is offering the lowest price.

Auction and fixed assignments mechanisms are interesting mechanisms and allow riders and drivers to negotiate the fair but the compensation of the platform provider has a considerable impact on the price paid by the riders and the revenue of the driver. For this reason, in our case study, we will omit this variable since our objective is to offer an additional service to the campus users and also to reduce traffic congestion around the university by persuading road users to share their cars. Eliminating such aspect, our model will focus on matching demands and supplies of rides among the students and the academic staff using a free application sponsored by the university. The objective will be, thus, to increase the overall welfare rather than maximize the platform's profit.

Besides these commercial applications of ridesharing that do not often reveal the tools they are implementing, there is, to the best of our knowledge, only a single attempt in the scientific literature of applying optimization tools for the ridesharing in a University campus (Amey, 2010). The application is concerned with the MIT campus in Massachusetts and considered a simplified variant of the ridesharing consisted of matching each driver with one single rider. The author has used a commercial software package CPLEX to solve the matching problem. Moreover, the author proposed a heuristic solution that selects the best rider-driver matching on the basis of a ranking procedure of the feasible matching using the VMT (Vehicle Miles Travelled) savings as a criterion.

Unlike Amey's paper, our work here consists in considering a more complex variant of the ridesharing problem since every vehicle can serve a driver-specified number of riders based on the available seats in her car. Moreover, since our preliminary results have recognized the limitations of CPLEX to solve real-life scenarios, here we propose a two-phase optimization approach that involves the solution of a constraint programming problem and a bin packing model. Our study appears to be a novel approach with promising applications.

\section{A Two-Phase Optimization Approach}

The performance of any ridesharing platform depends on the efficiency that one can achieve in solving the matching problem. We propose here an optimization approach based on a two-phase procedure. The first phase consists of generating all the feasible routes the drivers can perform in order to serve all the riders population. This step can be efficiently accomplished by solving a Constraint Programming (CP) model. Then, in the second phase we solve a bin-packing problem to select a subset of these feasible routes in order to define the optimal matching with the highest profit. The design of our approach is based on the following assumptions:

- Ignoring the time needed for picking up any rider.

- Ignoring the uncertainty due to traffic factors affecting the travel time between any two nodes.

- The drivers' average speed is considered to be constant.

The problem is defined over a transportation network represented by a directed graph whose nodes the drivers, and riders, locations. Moreover, the graph includes an additional node that 
represents Sultan Qaboos University (SQU) as the destination of all the participants. (Obviously, the definition of a similar approach for the outbound transportation from SQU to different locations is straightforward and does not present any further challenge). The notation to be used is as follows:
$P \quad$ Set of riders' pickup points
$\boldsymbol{L} \quad$ Set of drivers' locations
$\boldsymbol{S} \quad$ Final destination of all the participants, i.e., SQU
$\boldsymbol{D} \quad$ Set of drivers (i.e., available vehicles)
$\boldsymbol{A} \quad$ Set of riders asking for transportation service
$\boldsymbol{R} \quad$ Set of feasible ridesharing routes of all drivers
$\boldsymbol{C}_{\boldsymbol{k}} \quad$ Capacity of vehicle $k \in D$
$\boldsymbol{T}_{\boldsymbol{i}} \quad$ A driver specified parameter to indicate driver's $(i \in D)$ desired departure time before any deviation (it represents the arrival time when $i \in S$ )
$\boldsymbol{p}_{\boldsymbol{i} \boldsymbol{k}} \quad$ A parameter that takes value 1 if the starting location of driver $k$ is node $i$
$\boldsymbol{t}_{\boldsymbol{i j}} \quad$ Direct travelling time between locations $i$ and $j(i, j \in P \cup L \cup S)$
$\boldsymbol{b}_{\boldsymbol{i}} \quad$ Price to be paid by rider $i \in A$ for the ridesharing service

\subsection{Constraint Programming Model}

The decision variables of the CP model can be defined as follows:

$X_{i j k}$ : a binary variable that is 1 if vehicle $k$ travels directly from location $i$ to location $j$, and 0 , otherwise.

The CP model is defined to be:

$$
\begin{gathered}
\sum_{j \in P \cup S} X_{i j k}=p_{i k}, \quad \forall i \in L, \forall k \in D \\
\sum_{i \in P \cup L} X_{i j k}-\sum_{l \in P \cup S} X_{j l k}=0, \quad \forall j \in P, \forall k \in D \\
\sum_{k \in D} \sum_{i \in A} X_{i j k}=|A|, \quad \forall j \in S \\
\sum_{i \in L \cup P} \sum_{j \in P \cup L \cup S} X_{i j k} * t_{i j} \leq 2\left(T_{l}-T_{i}\right), \quad l \in S, \forall k \in D \\
\sum_{i \in P \cup L} \sum_{j \in P} X_{i j k} \leq C_{k}, \quad \forall k \in D \\
X_{i j k} \in\{0,1\} \forall i, j \in P \cup L \cup S, \forall k \in D \\
\text { "Subtour Elimination Constraints" }
\end{gathered}
$$

The set of constraints (1) ensures that each vehicle must start its route at the driver's location. Both the pickup and delivery of any rider must be made by the same vehicle. This is guaranteed by the set of constraints (2) that forces any vehicle that visits a pickup point $j$ to move to the next 
pickup point or to the delivery point (SQU). Constraints (3) ensure that all riders should reach their final destination (SQU). Constraints (4) set a maximum time spent by each driver on her route to SQU (the allowed deviation time to serve all the assigned rides has been chosen here to be twice the drive-alone time). Constraints (5) and (6) define the vehicles' capacity restrictions and the binary nature of all the variables, respectively, and finally constraints (7) against the presence of subtours in the feasible routes.

It is worth noting that while determining the set of all feasible routes $R$, our approach also computes the routing cost corresponding to every route generated by the $\mathrm{CP}$ model. We denote such a cost as $h_{k r}$, for every route $r \in R$ and every vehicle $k$.

\subsection{Bin Packing Based Model}

For the selection of the subset of routes to be accomplished by the drivers, we use a Bin Packing (BP) based model that uses a set of binary variables $Y_{k r}$ each taking the value 1 if driver $k$ is assigned to route $r$, and 0 , otherwise. The BP problem can be described as follows:

$$
\begin{gathered}
\operatorname{Max} \sum_{r \in R} \sum_{k \in D}\left(\sum_{i \in A} b_{i} E_{i r}-h_{k r}\right) \cdot Y_{k r} \\
\sum_{r \in R} Y_{k r} \leq 1, \quad \forall k \in D \\
\sum_{k \in D} \sum_{r \in R} E_{i r} Y_{k r} \leq 1, \quad \forall i \in A \\
Y_{k r} \in\{0,1\}, \quad \forall r \in R, \quad \forall k \in D,
\end{gathered}
$$

in which $E_{r k}(i \in P$ and $r \in R$ ) represents the binary route-rider assignment (parameter) that takes the value 1 only if rider's location $i$ is involved in route $k$, and 0 , otherwise.

The objective (8) is to maximize the matching profit defined as the amount to be paid by all the riders minus the total travelling cost over all the routes to be performed by all the drivers. Constraints (9) ensure that each vehicle is assigned at most to one route. Constraints (10) guarantee that each rider is served by at most one of the feasible routes. Finally, constraints (11) force the decision variables to be binary. Our model clearly does not involve the "obligation to serve" rule; any matching will oblige to only the profit maximization criterion.

The BP model (8)-(11) turns to be a linear combinatorial optimization model which is known to be NP-Hard. Even though solving very large real-life problems can be a challenging task, for our specific application to SQU, general purpose software packages (such as Lingo, Matlab, GAMS and Cplex) can be used in order to solve moderate size problems. 


\section{Decision Support System}

In this section we will describe the details of our platform system including the payment scheme and the re-optimization approach when a new customer is included in any of the drivers' tour. Even though the payment scheme is very important to motivate drivers to share their cars with riders, it is a challenging task to design a dynamic system that allows the incorporation of new riders in the drivers' routes. For this purpose, a decision support system is required to allow new riders to appear automatically in the system, and get a convenient driver assigned. For this purpose, the demonstrative decision support system implemented for Sultan Qaboos University consists of a web application as well as a smartphone application built in the form of a database incorporating dynamic receive and retrieve functionality.

\subsection{Design of the Payment Scheme}

Our suggested payment scheme in the city of Muscat is using a fixed rate of (0.125 OMR) per pick up if the total deviation from the main route is less than 10 minutes. However, if the deviation is more than 10 minutes, then the increment will be $(0.025$ OMR) for every extra minute. The total pay curve will be as shown in Fig. 1. Our approach has also simulated the behavior of the price with respect to the market size by considering three possible scenarios (Fig. 2) of low, average and high cases. We have assumed that these three scenarios can occur with probabilities of $0.15,0.7$ and 0.15 , respectively.

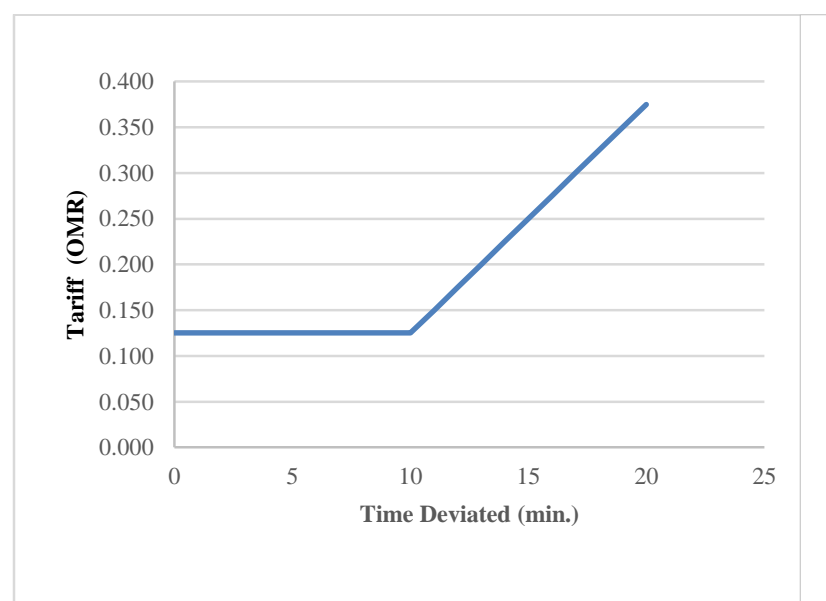

Figure 1. Payment scheme

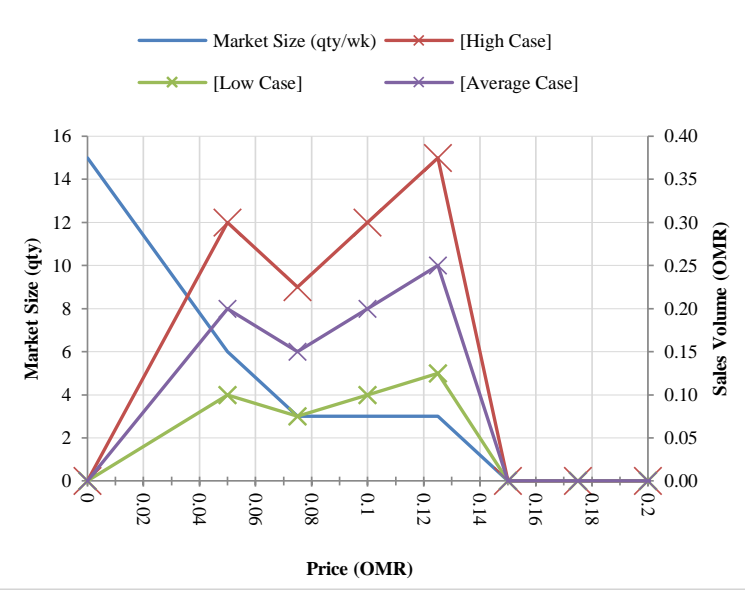

Figure 2. Market size vs. price

Figs. 1 and 2 show that the maximum Expected Monetary Value (EMV) can be achieved at rate of 0.125 OMR per pickup. While comparing the other options for tariff, the market size reaches its maximum when the price is 0 OMR and drops as the price increases; any tariffs above 0.150 OMR does not attract riders anymore.

\subsection{Linking the Optimization Model with the Decision Support System}

The Data System Design (DSD) is the process of designing the tables of inputs and outputs for the display in the dashboard. These consist of either inputs data of riders and drivers, or the matching solutions of the model. The DSD is composed of three elements: tables design, form 
design, and report design. The inputs register is based on three main tables: Request to Pick (RTP), Drivers, and Routes. The form design for RTP automatically sets the value for Rider Number, and the Riders (the users) can input the departure location (selected from a defined list of departure locations), desired arrival time, and contact number. After submission, the rider will receive a message of acknowledgement. The form design for Drivers automatically sets the value for Driver ID, and the Drivers (the users) can input the departure location, departure time, desired arrival time, vehicle capacity, preferred route, and contact number.

The departure location, departure time, and desired arrival time are passed on exit of submission as parameters for the riders/drivers matching. The driver registration gives a summary of the registered information which turns to be useful for the driver to display potential riders.

\subsection{Information Flow and Insertion Heuristic}

The flow of information is very important for the model to function as intended. The data system will have two inputs, where riders register their RTP and drivers register their availability. Then, the drivers can have access to the list of riders assigned to them in the system. Fig. 3 illustrates the information flow in the data system. Given the dynamic nature of the problem, a new request may arrive at the system after solving the matching problem but before ending the implementation of the riding trips. In this case, the platform will use an insertion procedure in order to investigate if it is possible to define a feasible rider-driver assignment having the minimum Expected Delay (ED). Such a heuristic is based on the best-of-the-worst criterion that modifies the route of one single driver only without re-optimizing the whole matching. From this point of view, the whole approach can be seen as two-stage recourse procedure in which the first stage consists of the initial matching resulting from solving the mathematical model and the recourse second stage consists of inserting additional riders within the existing routes.

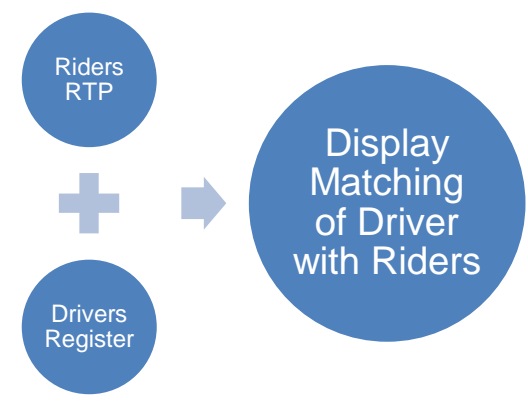

Figure 3. Information flow in the data system

\section{Decision Support System Experiments}

The experiments related to our DSS have been conducted on a test problem including a set of data registered in the system having 12 Riders who requested a pickup, and 8 Drivers available to share their own cars. Such experiments have the objective of validating our approach. These test 
instances have been generated to represent realistic scenarios, since on one side real data are not available yet and on the other side no standard test problems are available for these kinds of problems.

The data registered in the system are as summarized in Table 1 for RTPs and Table 2 for Drivers. Since the model is to be used for a university campus, the destination for riders is always to and from the campus.

Table 1. Data registered for RTP

\begin{tabular}{|llllll|} 
RiderNo & Dept_loc & DAT_R & Contact & PickedUp & Tariff \\
\hline RD001 & Al Khoudh 6 & 8 & 98765432 & FALSE & 0 \\
\hline RD002 & Al Maabila & 10 & 98765432 & FALSE & 0 \\
\hline RD003 & Al Khoudh 6 & 12 & 11223344 & FALSE & 0 \\
\hline RD004 & Al Mawaleh South & 14 & 11223344 & FALSE & 0 \\
\hline RD005 & Al Mawaleh South & 14 & 11223344 & FALSE & 0 \\
\hline RD008 & Al Mawaleh North & 12 & 98465651 & FALSE & 0 \\
\hline RD009 & Al Maabila & 8 & 86416846 & FALSE & 0 \\
\hline RD010 & Al Mawaleh South & 10 & 68418942 & FALSE & 0 \\
\hline RD011 & Al Amirat & 8 & 68146349 & FALSE & 0 \\
\hline RD012 & Al Mawaleh North & 16 & 71897894 & FALSE & 0 \\
\hline
\end{tabular}

Table 2. Data registered for drivers

\begin{tabular}{|lllllll|} 
DRV_ID & Dept_Loc & Dept_Time & DAT & Veh_Cap & Pref_Route & Contact \\
\hline DRV001 & Al Khoudh & 7.25 & 8 & 3 & 1 & 12345678 \\
\hline DRV002 & Al Maabila & 7.5 & 8 & 2 & 4 & 12345678 \\
\hline DRV003 & Al Khoudh 6 & 7 & 8 & 2 & 3 & 12346579 \\
\hline DRV004 & Al Mawaleh North & 11 & 12 & 2 & 4 & 65685165 \\
\hline DRV005 & Al Amirat & 7 & 8 & 1 & 2 & 68146384 \\
\hline DRV006 & Al Maabila & 7 & 8 & 3 & 2 & 86419849 \\
\hline DRV007 & Al Mawaleh South & 13 & 14 & 2 & 1 & 19643943 \\
\hline DRV008 & Al Mawaleh North & 9 & 10 & 3 & 4 & 13684368 \\
\hline
\end{tabular}

The model is executed to match the drivers with potential riders by means of sub-optimizing each driver parcours rather than the whole system due to its dynamic nature. The dynamic nature of the system implies that any insertion of new data to the system can be directly optimized even if the driver has already approved of the riders for pickup. Figs. 4, 5, and 6 illustrate examples of several drivers/riders matchings. It is clear that "driver 003", for example, will make departure for "Al Khoud" as destination and will pick up riders "RD1" and "RD03" and will have 30 minutes delay before reaching the University Campus. 


\begin{tabular}{l} 
Driver Departure Location Al Khoudh 6 \\
Driver ID DRV003 \\
Drivers Departure Time 7 \\
Vehicle Capacity 2 \\
Driver's Route 3 \\
Driver Contact Number 12346579 \\
Rider ID RD001 \\
Rider Location Al Khoudh 6 \\
Rider Contact 98765432 \\
Expected Delay (min) For Driver 10 \\
RTP Tariff 0 \\
To Be Picked? No \\
\hline \\
Driver Departure Location Al Khoudh 6 \\
Driver ID DRV003 \\
Drivers Departure Time 7 \\
Vehicle Capacity 2 \\
Driver's Route 3 \\
Driver Contact Number 12346579 \\
Rider ID RD003 \\
Rider Location Al Khoudh 6 \\
Rider Contact 11223344 \\
Expected Delay (min) For Driver 10 \\
RTP Tariff 0 \\
To Be Picked? No \\
\hline
\end{tabular}

Figure 4. Matching for DRV003
Driver Departure Location Al Amirat

Driver ID DRV005

Drivers Departure Time 7

Vehicle Capacity 1

Driver's Route 2

Driver Contact Number 68146384

Rider ID RD011

Rider Location Al Amirat

Rider Contact 68146349

Expected Delay (min) For Driver 15

RTP Tariff 0

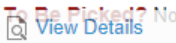

Figure 5. Matching for DRV005

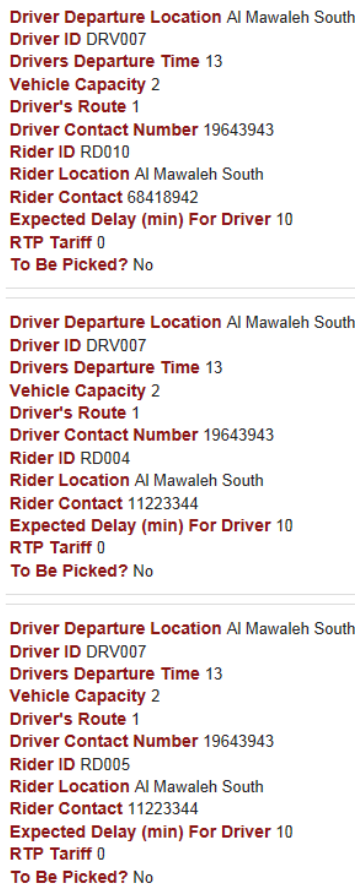

Figure 6. Matching for DRV007

The CPU time needed to solve this illustrative example is in the order to a few seconds only. Even though solving real-life applications may be more challenging, we are confident that our DSS is able to handle large-scale instances characterized by a big number of RTPs and available drivers. However, we are conscious that solving the constraint programming model to generate all the feasible routes may be a bottleneck in the platform once a given threshold number of agents is reached. In that case, it is possible to develop a truncated constraint programming procedure that generates not necessarily all the feasible routes but is satisfied with the promising ones only. The truncating decision may be based on several criteria such as maximum detour time or distance, minimum profit, etc. The development of this idea is left for a future investigation.

\section{Conclusions}

Introducing ridesharing in the Sultan Qaboos University (SQU) campus to a large extent is needed to reduce traffic congestion and pollution problems. While in industrialized countries (such as Australia, UK and USA) ridesharing is widely diffused, in developing countries this phenomenon is limited to a reduced social network of friends, parents and colleagues. The model developed for SQU campus can be extended to other campuses and cities in Oman to reduce traffic congestion, air pollution and fuel consumption. For this purpose, it will be necessary to develop a ridesharing platform that can be used at a commercial scale. Thus, a SWOT analysis of the potentials is discussed below. 


\begin{tabular}{|c|c|c|}
\hline 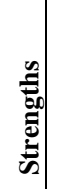 & $\begin{array}{ll}\text { - } & \text { Mark } \\
\text { - } & \text { Rela } \\
\text { - } & \text { No p }\end{array}$ & $\begin{array}{l}\text { r the drivers } \\
\text { nethod }\end{array}$ \\
\hline & $\begin{array}{l}\text { - No ridesharing platform } \\
\text { implemented so far in SQU } \\
\text { - Many new students do not have } \\
\text { means of transportation }\end{array}$ & $\begin{array}{l}\text { not pay } \\
\text { delay driver mor } \\
\text { detour for rides }\end{array}$ \\
\hline
\end{tabular}

Launching a commercial activity as a ridesharing provider can be very profitable, but for this case, there is not yet a clear scheme on how much each rider or/and driver would pay to the service provider. The only transaction is so far limited to the direct rider-to-driver payment. The provider's share can become either an encouraging or an inhibitive factor for the success of ridesharing in Oman. The threshold of acceptability depends on the country and the absence of any previous experience in this context in Oman makes it a crucial factor that may require not only quantitative expertise but also socio-economical studies.

Besides these technical issues, the spread of ridesharing in Oman should overcome several social obstacles. First, gender can be a serious constraint since the local culture is based on gender segregation. According to local traditions, in Oman a woman not accompanied by a family member cannot join a car driven by an unknown man. Another issue can be the local habits of relying on cars in any movement and the large diffusion of private cars (together with the relatively low cost of fuel).

Finally, having the governmental authorization for a new platform to operate in Muscat is also a hurdle since taxi drivers have the monopoly of ground transportation and ridesharing transportation modality will probably stimulate new challenges and make their business difficult to survive. Involving taxi drivers in the ridesharing business has been adopted by Zimcar platform in the USA, and this option can be adopted in further studies to encourage a large diffusion of ridesharing in Muscat. Probably, in absence of an adequate public transportation network, offering lower prices, flexible and available rides can persuade car owners to give up the luxury to use their own cars and hence reduce traffic congestion.

\section{References}

[1] Agatz E., Savelsbergh M. and Xing W. (2012), Optimization for dynamic ridesharing: A review. European Journal of Operational Research 223(2), 295-303

[2] Al-Riyami M., Ismail N., Al-Azri A. and Triki C. (2016), A Matching Optimization Model for the Dynamic Ridesharing in a University Campus. Proceedings of the International Conference on Applied Mechanics and Industrial Systems, 6-8 December, Muscat.

[3] Amey A. (2010), A Proposed Methodology for Estimating Rideshare Viability within an Organization, applied to the MIT Community. Working paper, Massachusetts Institute of Technology. http://ridesharechoices.scripts.mit.edu/home/wpcontent/papers/AAmey_11.2585_TRB2011_RideshareModel_Ver1.pdf

[4] Asghari M., Deng D., Shahabi C., Demiryurek U. and Li Y. (2016), Price-aware real-time ridesharing at scale: an auction-based approach. Proceedings of the 24th ACM 
International Conference on Advances in Geographic Information Systems.

[5] Brownstone D. and Golob T.F. (1992), The effectiveness of ridesharing incentives: Discrete-choice models of commuting in Southern California, Regional Science and Urban Economics, 22, 5-24

[6] Cook, J.L. and Ramirez-Marquez, J.E. (2007), Two-terminal reliability analyses for a mobile ad hoc wireless network, Reliability Engineering and System Safety, 92(6), 821829.

[7] Locks, M.O. (1985), Recent developments in computing of system-reliability, IEEE Transactions on Reliability, R-34(5), 425-436.

[8] Xue, J. (1985), On multistate system analysis, IEEE Transactions on Reliability, R-34, 329-337.

[9] Yeh, W.C. (2002a), Search for all $d$-Mincuts of a limited-flow network, Computers and Operations Research, 29(13), 1843-1858.

[10] Yeh, W.C. (2002b), A new approach to the $d-M C$ problem, Reliability Engineering and System Safety, 77(2), 201-206.

[11] Kleiner A., Nebel B., and Ziparo V. A. (2011), A mechanism for dynamic ridesharing based on parallel auctions. IJCAI'11 Proceedings of the Twenty-Second international joint conference on Artificial Intelligence, pp. 266-272

[12] Morency C., (2007), The ambivalence of ridesharing, Transportation, 34(2), 239-253

[13] Nisan N., Roughgarden T., Tardos E. and Vazirani V. V. (2007), Algorithmic Game Theory, New York, NY, USA: Cambridge University Press.

[14] Oxford Business Group - Oman (2012), Report: www.oxfordbusinessgroup.com/country/Oman

[15] World population Review (2017), Report: http://worldpopulationreview.com/countries/oman-population/

[16] Zhao D., Zhang D., Gerding E. H., Sakurai Y. and Yokoo M. (2014), Incentives in Ridesharing with deficit control, Proceedings of the 2014 International Conference on Autonomous agents and multi-agent systems (AAMAS), pp. 1021-1028.

[17] Zhu G.-Q., Tong G.-J. and Dai L.-L. (2008), Analysis of urban road congestion pricing based on game theory, Proceedings of the 15th International Conference on Management Science and Engineering, pp. 1693-1697 